\title{
Plasma fatty acid changes following consumption of dietary oils containing n-3, n-6, and n-9 fatty acids at different proportions: preliminary findings of the Canola Oil Multicenter Intervention Trial (COMIT)
}

Vijitha K Senanayake ${ }^{1,6}$, Shuaihua Pu', David A Jenkins², Benoît Lamarche ${ }^{3}$, Penny M Kris-Etherton ${ }^{4}$, Sheila G West ${ }^{4,5}$, Jennifer A Fleming ${ }^{4}$, Xiaoran Liu ${ }^{4}$, Cindy E McCrea ${ }^{5}$ and Peter J Jones ${ }^{*^{*}}$

\begin{abstract}
Background: The Canola Oil Multicenter Intervention Trial (COMIT) was a randomized controlled crossover study designed to evaluate the effects of five diets that provided different oils and/or oil blends on cardiovascular disease (CVD) risk factors in individuals with abdominal obesity. The present objective is to report preliminary findings on plasma fatty acid profiles in volunteers with abdominal obesity, following the consumption of diets enriched with n-3, n-6 and n-9 fatty acids.

Methods: COMIT was conducted at three clinical sites, Winnipeg, Manitoba, Canada, Québec City, Québec, Canada and University Park, Pennsylvania, United States. Inclusion criteria were at least one of the followings: waist circumference ( $\geq 90 \mathrm{~cm}$ for males and $\geq 84 \mathrm{~cm}$ for females), and at least one other criterion: triglycerides $\geq 1.7 \mathrm{mmol} / \mathrm{L}$, high density lipoprotein cholesterol $<1 \mathrm{mmol} / \mathrm{L}$ (males) or $<1.3 \mathrm{mmol} / \mathrm{L}$ (females), blood pressure $\geq 130 \mathrm{mmHg}$ (systolic) and/or $\geq 85 \mathrm{mmHg}$ (diastolic), and glucose $\geq 5.5 \mathrm{mmol} / \mathrm{L}$. Weight-maintaining diets that included shakes with one of the dietary oil blends were provided during each of the five 30-day dietary phases. Dietary phases were separated by four-week washout periods. Treatment oils were canola oil, high oleic canola oil, high oleic canola oil enriched with docosahexaenoic acid (DHA), flax oil and safflower oil blend, and corn oil and safflower oil blend. A per protocol approach with a mixed model analysis was decided to be appropriate for data analysis.

Results: One hundred and seventy volunteers were randomized and 130 completed the study with a dropout rate of $23.5 \%$. The mean plasma total DHA concentrations, which were analyzed among all participants as a measure of adherence, increased by more than $100 \%$ in the DHA-enriched phase, compared to other phases, demonstrating excellent dietary adherence.
\end{abstract}

Conclusions: Recruitment and retention strategies were effective in achieving a sufficient number of participants who completed the study protocol to enable sufficient statistical power to resolve small differences in outcome measures. It is expected that the study will generate important data thereby enhancing our understanding of the effects of n-3, n-6, and n-9 fatty acid-containing oils on CVD risks.

Trial registration: ClinicalTrials.gov NCT01351012.

Keywords: Cardiovascular diseases, Metabolic syndrome, Plasma fatty acids, Serum lipids, Lipoproteins, Canola oil, DHA, Randomized controlled clinical trial

\footnotetext{
* Correspondence: peter_jones@umanitoba.ca

${ }^{1}$ Richardson Centre for Functional Foods and Nutraceuticals, University of Manitoba, 196 Innovation Drive, SmartPark, Winnipeg, MB R3T 6C5, Canada Full list of author information is available at the end of the article
} 


\section{Background}

It is well established that decreasing dietary saturated fatty acids (SFA) reduces the risk of cardiovascular disease (CVD) [1]. Current dietary recommendations advise that unsaturated fatty acids should replace SFA with little guidance provided about the precise amounts that should be substituted [2]. Epidemiological evidence indicates various fatty acids classes including n-9 monounsaturated (MUFA), n-3 and n-6 polyunsaturated fatty acids (PUFA) as replacements for SFA with more health benefits [3-8]. Despite the large body of research evaluating the effects of different fatty acid classes, studies that systematically and simultaneously compare multiple fatty acid classes have not been conducted. Moreover, a need exists to evaluate additional biomarkers, beyond blood lipids and/or lipoproteins, that better estimate risk of clinical outcomes [9], and to achieve sufficiently large sample sizes in order to resolve modest differences and high variability in endpoint measurements. Additionally, significant knowledge gaps remain in our understanding of the effects of, and mechanisms underpinning the action of, the various fatty acid classes on risk factors for chronic diseases.

One such controversy is the debate surrounding $\alpha$ linolenic acid (ALA). Whether its effects are dependent on its conversion to longer chain n-3 fatty acids [10-12] needs to be better substantiated. The relative efficacy of different classes of PUFA, specifically linoleic acid (LA) [13], ALA and docosahexaenoic acid (DHA), in modulating inflammatory processes and endothelial function also remains to be elucidated $[14,15]$. The reported undesirable effects of LA [16] are of particular concern. A direct comparison of dietary n- 6 with n-3 fatty acids on inflammatory biomarkers and endothelial function would also be helpful in clarifying these issues. Since inflammation directly impacts endothelial function and the progression of atherosclerosis $[17,18]$, endothelial function measurements would serve as useful biomarkers of CVD risk.

Abdominal obesity and insulin resistance are criteria for metabolic syndrome [19,20]. Dietary fat has been implicated in the pathogenesis of this syndrome via ectopic adipose tissue deposition [21,22]. Different classes of fatty acids appear to have different effects on body fat accretion [23-26]. Consequently, a need also exists to evaluate the effects of various fatty acid classes on body composition and body fat distribution.

Based on the above rationale, a multicenter randomized clinical trial was designed to evaluate the biological effects of conventional canola oil, high oleic canola oil, DHAenriched high oleic canola oil, a blend of flax oil with safflower oil, and a blend of corn oil and safflower oil. The contrast selected allowed for comparisons of the effects of oils rich in n-9 versus n-3 and n-6 oil blends.
Circulating lipids and/or lipoproteins, inflammatory biomarkers, endothelial function and body composition were evaluated after each dietary treatment period. Additionally, mechanistic assessments including reverse cholesterol transport, stable isotope fatty acid conversion, and fatty acid desaturase genetic variation analysis were also carried out. Consequently, the purpose of this study was to comprehensively investigate the effects of major fatty acid classes on biomarkers of chronic disease at a mechanistic level. This paper describes the protocol and subject recruitment experience for the COMIT study and presents plasma fatty acid data.

\section{Methods}

\section{Study design}

COMIT was designed as a five-phase randomized controlled double-blind crossover clinical trial. One-hundred and seventy subjects ( $\mathrm{n}=95$ females) aged 18 years or older were selected with at least one metabolic syndrome clinical criterion. Participants were recruited at two participating centers in Canada, including the Richardson Centre for Functional Foods and Nutraceuticals (RCFFN) at the University of Manitoba in Winnipeg $(n=69)$, and the Institute on Nutrition and Functional Foods (INAF) at Laval University in Québec City $(\mathrm{n}=58)$, as well as one clinical institution in the United States: the Departments of Nutritional Sciences and Biobehavioral Health, Pennsylvania State University, University Park $(n=43)$. The Risk Factor Modification Centre (RFMC) at St. Michael's Hospital in Toronto, Canada participated in the sample analyses.

\section{Study recruitment and retention}

Recruitment was conducted using radio and newspaper advertisements, direct mailings, community meetings, and advertised information sessions as depicted in Table 1. On first contact, volunteers completed a questionnaire to determine their eligibility for the study. Qualifying participants were instructed to provide a fasting blood sample at their convenience. Eligible participants were selected based on the inclusion criteria and exclusion criteria described below. The flow of participants from initial contact to completion is given in Figure 1.

At RCFFN in Winnipeg, a social program for study participants termed 'Club Richardson' was initiated for the purpose of fostering interaction and cooperation among participants to enhance their retention and compliance. Nutrition-related forums and outings were conducted, and tickets to hockey and soccer games were provided. Specific events were conducted to coincide with celebrations such as Christmas. Wine and cheese events were also held during the washout periods. Cards and flowers were gifted to coincide with birthdays. Similar retention strategies were used at the other two clinical centers. Personal interaction 
Table 1 Recruitment strategies employed at participating clinical sites

\begin{tabular}{|c|c|}
\hline Clinical sites & Recruitment tools \\
\hline \multirow[t]{5}{*}{ Winnipeg (Manitoba, Canada) } & $\begin{array}{l}\text { Direct mail outs to neighborhoods } \\
\text { in the City of Winnipeg }\end{array}$ \\
\hline & University of Manitoba email system \\
\hline & Radio advertisements \\
\hline & $\begin{array}{l}\text { Recruitment seminars at community } \\
\text { centers at various localities }\end{array}$ \\
\hline & University of Manitoba bulletin boards \\
\hline \multirow[t]{3}{*}{ Québec City (Québec, Canada) } & $\begin{array}{l}\text { Institute of Nutrition and Functional } \\
\text { Foods (INAF) mailing list }\end{array}$ \\
\hline & Laval University email system \\
\hline & Newspaper advertisements \\
\hline \multirow{4}{*}{$\begin{array}{l}\text { University Park (Pennsylvania, } \\
\text { United States) }\end{array}$} & Pennsylvania State University email \\
\hline & Flyers \\
\hline & Local newspaper advertisements \\
\hline & Television advertisements \\
\hline
\end{tabular}

was a high priority among the clinical staff and study participants at all centers.

\section{Inclusion criteria}

Initially, discussions about the eligibility criteria of the study were based on the following three parameters; 22 to $32 \mathrm{~kg} / \mathrm{m}^{2}$ body mass index (BMI), plasma triglyceride $>1.7 \mathrm{mmol} / \mathrm{L}$, and waist to hip ratio of $>0.85$. When the protocol was finalized, it was decided that focus should be placed on waist circumference as the primary inclusion criterion because of the relationship between visceral fat and endothelial function [27]. At the beginning of the study, the Adult Treatment Panel III (ATP III) metabolic syndrome criteria for waist circumference $(>102 \mathrm{~cm}$ for men and $>88 \mathrm{~cm}$ for women) were followed [28]. As the trial progressed, the International Diabetes Federation (IDF) metabolic syndrome criteria for waist circumference ( $\geq 94 \mathrm{~cm}$ for men and $\geq 80 \mathrm{~cm}$ for women) were adopted [29] to identify individuals in the initial stages of abdominal obesity who might benefit from dietary intervention. This change occurred at the same time in all centers and did not

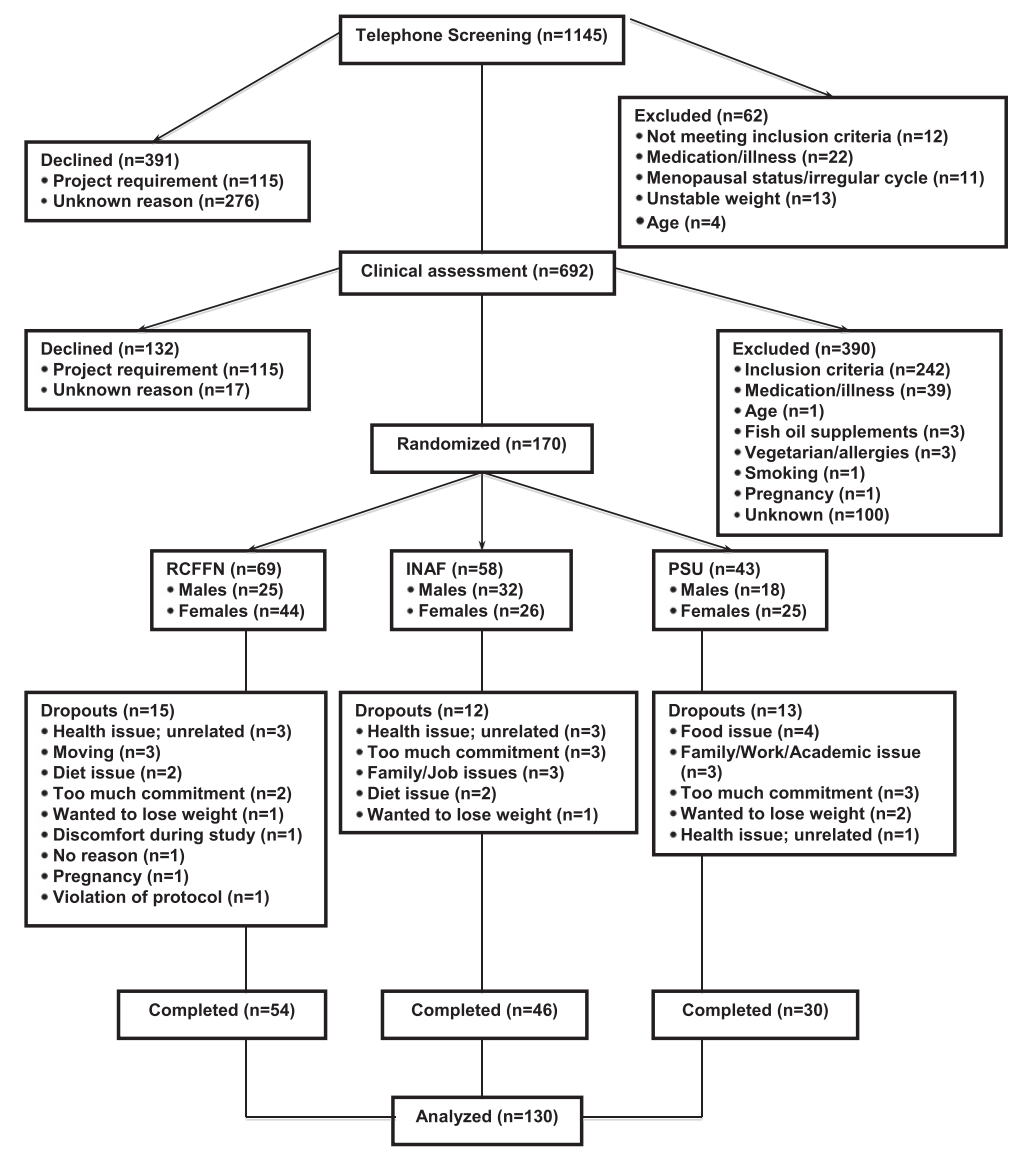

Figure 1 Flow of participants in the COMIT study. The participant flow through each step of the COMIT recruitment, screening and study protocol process. RCFFN, Richardson Centre for Functional Foods and Nutraceuticals. INAF, Institute on Nutrition and Functional Foods; PSU, Pennsylvania State University. 
affect the subjects already recruited as the waste circumference cutoff values were lowered. Other inclusion criteria were triglyceride level (TG) $\geq 1.7 \mathrm{mmol} / \mathrm{L}$, high density lipoprotein cholesterol level (HDL) $<1 \mathrm{mmol} / \mathrm{L}$ (males) or $<1.3$ $\mathrm{mmol} / \mathrm{L}$ (females), blood pressure $\geq 130 \mathrm{mmHg}$ (systolic) and/or $\geq 85 \mathrm{mmHg}$ (diastolic) and glucose level $\geq 5.5$ $\mathrm{mmol} / \mathrm{L}$. Participants were required to meet at least the waist criterion or any one of the other inclusion criteria in order to be eligible.

\section{Exclusion criteria}

Individuals with thyroid disease (unless controlled by medication), diabetes mellitus, kidney disease, liver disease, current smokers, or those consuming more than two alcoholic drinks per week, were excluded from the study. Individuals taking medications known to affect lipid metabolism or endothelial function (including aspirin or other non-steroidal anti-inflammatory drugs), cholestyramine, colestipol, niacin, clofibrate, gemfibrozil, probucol, or 3-hydroxy-3-methyl-glutaryl-CoA (HMG-CoA) reductase inhibitors were excluded. In addition, individuals were not allowed to participate if they were unwilling to stop taking any supplements at least two-weeks prior to the study. Creatinine, serum glutamate pyruvate transaminase (SGPT), serum glutamic oxaloacetic transaminase (SGOT), alkaline phosphatase (ALKP), lactate dehydrogenase (LDH), glucose and lipid profiles that included total cholesterol (TC), TG, low density lipoprotein (LDL), and HDL were also analyzed at screening.

\section{Ethical considerations}

The study protocols (with ethical considerations) were reviewed and approved by research ethics boards at each participating institution, including the Biomedical Ethics Board at the University of Manitoba, Comité sectoriel d'éthique de la recherche en sciences de la santé de l'Université Laval, and the Institutional Review Board at the Pennsylvania State University. The trial was registered at clinicaltrials.gov under the registration number NCT01351012. Written informed consent was obtained after an initial interview with each participant. The interview included a full description of the study and a discussion of the compliance issues and study expectations.

\section{Randomization}

Random permutations of the five treatments were created by the study director at the main site using a random number generator to ensure that all the subjects received treatments in a balanced and random order. Sequences were coded for the purpose of blinding the investigators and the subjects. Coded sequences were kept in sealed envelopes at the Winnipeg center and were assigned to subjects by study coordinators at respective sites as they joined the study across the three centers.

\section{Study diets}

Participants consumed a controlled weight-maintenance, full-feeding diet with a fixed macronutrient composition (35\% fat, 50\% carbohydrate, $15 \%$ protein, <200 mg cholesterol, 35 to $40 \mathrm{~g}$ fiber) for five treatment periods. They were provided with three meals and two snacks a day using a seven day rotating menu for the complete duration of each dietary phase. Each of five treatment phase extended four 4 weeks and separated by four-week washout intervals, although ten volunteers took shorter washout periods of two to four weeks at the PSU site. Treatment oils were provided as a shake-style beverage, divided in two equal doses to be consumed at breakfast and supper. Participants were instructed to consume one of their meals under supervision on site. Treatment oils were: 1) conventional canola oil (Canola), 2) high oleic canola oil (CanolaOleic), 3) $85 \%$ high oleic canola and 15\% DHA oil blend (CanolaDHA), 4) 60\% flax oil and $40 \%$ safflower oil blend (FlaxSaff), and 5) 25\% corn oil and $75 \%$ safflower oil blend (CornSaff). The amount of treatment oil and/or oil blend was determined based on the calculated energy requirements of the participants, with the $3000 \mathrm{Kcal}$ intake targeted to consume $60 \mathrm{~g}$ oil per day, and the 1800 and $2400 \mathrm{Kcal}$ groups to consume 36 and $48 \mathrm{~g}$ per day, respectively. Oils were weighed to the nearest gram and added to the shakes. Meals and shakes were prepared in the metabolic kitchen where kitchen staff and clinical coordinators were blinded to the treatments. Meals and shakes were provided in cold packs for meals consumed offsite. These take-out meals were provided to participants at each center during their daily visit. Weekend meals and shakes were delivered to the participants' place of residence or were dispensed at the feeding site on Fridays.

A subgroup of participants in Winnipeg prepared their own meals under the supervision of a trained individual. This was possible due to personal cultural practices involving the use of a common kitchen to prepare community meals. Treatment shakes were prepared at the RCFFN and delivered to the community every two to three days.

Subjects were instructed to consume only the prepared meals and to limit their consumption of alcohol or caffeinated beverages. Diets were planned for every subject according to their energy requirements and were nutritionally adequate. Energy requirement was calculated using the Mifflin-St Jeor equation [30]. During each study period body weights were monitored, and if subjects gained or lost weight energy adjustments were made. Subjects were given a diet that met the Canadian and United States recommended nutrient intakes. The nutrient content of the diet was analyzed using FOOD PROCESSOR (ESHA Research, Salem, Oregon, United 
States) to verify macronutrient composition and assure micronutrient adequacy.

\section{Compliance}

Compliance during the trial was determined based on the remaining amounts of treatment shakes in pre-packaged food packs intended for off-site consumption, and by a weekly dietary questionnaire on dietary deviations which ensured that macro- and micronutrient intakes remained unchanged. Subjects were instructed to consume at least one meal on site, further ensuring compliance. These observations were further confirmed by expected changes in plasma fatty acid profiles at the end of each dietary period. For example, a greater than two-fold baseline to endpoint increase in serum DHA was expected during the CanolaDHA dietary period.

\section{Intervention}

Four oils with different n-9: n-6: $n-3$ ratios were selected as the intervention; conventional canola oil (6:2:1), high oleic canola oil (31:6:1), DHA-enriched high oleic canola oil (6:1:1), and a flax oil and safflower oil blend (1:1:1). The fatty acid composition varied noticeably among treatment oils (Table 2), allowing comparison of the effects of n-3 fatty acids, ALA and DHA, n-6 fatty acid, LA, and n-9 fatty acid, oleic acid.

\section{Comparison dietary fat blend}

An oil blend of corn (25\%) and safflower (75\%) with n-9: n-6: n-3 ratio of 6:25:0.1 was used as the comparison instead of a high saturated fat blend. Since the effects of saturated fat have been well established it was deemed unnecessary to include it as a control. Moreover, we previously examined the effects of high oleic canola and flax oil compared to a diet high in saturated fat [23,31]. Additionally n- 6 fatty acids, which are abundant in the comparison oil blend, are considered essential and healthy, however the concept of the n- 6 to $n-3$ ratio and the effects on inflammation merits re-examination $[13,16]$. Using n-6 rich oil enabled us to investigate these concepts.

\section{Outcome measures}

Endothelial function was identified as the primary endpoint based on our rationale of evaluating emerging CVD risk factors beyond traditional lipid biomarkers for CVD. Secondary endpoints and laboratory analyses are presented in "List of secondary endpoints analyzed in the COMIT study" section. All centers followed identical procedures on all the measurements to minimize the variation. The peripheral arterial tonometry (PAT) technique was utilized to measure endothelial function. By using an Endo-PAT device (Itamar Medical, Caesarea, Israel), this technique measures the reactive hyperemia which is significantly correlated with endothelium-dependent vasodilatation [32].
Table 2 Fatty acid composition of dietary oils (\%) ${ }^{a}$

\begin{tabular}{|c|c|c|c|c|c|}
\hline Fatty acids & Canola & CanolaOleic & $\begin{array}{c}\text { CanolaDHA } \\
(85: 15)\end{array}$ & $\begin{array}{c}\text { FlaxSaff } \\
(60: 40)\end{array}$ & $\begin{array}{c}\text { CornSaff } \\
(25: 75)\end{array}$ \\
\hline c4:0 & 0 & 0 & 0 & 0 & 0 \\
\hline c6:0 & 0 & 0 & 0 & 0 & 0 \\
\hline c8:0 & 0 & 0 & 0 & 0 & 0 \\
\hline c10:0 & 0 & 0 & 0 & 0 & 0 \\
\hline c12:0 & 0.09 & 0.06 & 0.07 & 0 & 0 \\
\hline c14:0 & 0.07 & 0.07 & 0.78 & 0 & 0.01 \\
\hline c16:0 & 4.06 & 3.66 & 5.25 & 4.90 & 5.86 \\
\hline c17:0 & 0.07 & 0.09 & 0.17 & 0 & 0.02 \\
\hline c18:0 & 1.83 & 1.83 & 1.70 & 3.17 & 1.90 \\
\hline c20:0 & 0.65 & 0.65 & 0.57 & 0 & 0.11 \\
\hline C22:0 & 0.31 & 0.31 & 0.30 & 0 & 0 \\
\hline c24:0 & 0.18 & 0.18 & 0.15 & 0 & 0 \\
\hline Total SFA & 7.26 & 6.85 & 8.99 & 8.07 & 7.90 \\
\hline c14:1 & 0 & 0 & 0.01 & 0 & 0 \\
\hline c16:1 & 0.25 & 0.22 & 0.21 & 0 & 0.03 \\
\hline c17:1 & 0.12 & 0.20 & 0.17 & 0 & 0 \\
\hline c18:1 & 58.61 & 71.47 & 63.25 & 17.86 & 17.60 \\
\hline c20:1 & 1.22 & 1.20 & 1.03 & 0 & 0.03 \\
\hline C22:1 & 0.05 & 0.07 & 0.06 & 0 & 0 \\
\hline Total MUFA & 60.25 & 73.16 & 64.73 & 17.86 & 17.66 \\
\hline c18:2 n-6 & 19.54 & 14.74 & 12.74 & 37.47 & 69.34 \\
\hline c18:3 n-3 & 9.76 & 2.30 & 1.96 & 31.98 & 0.29 \\
\hline$c 20: 4 n-6$ & 0 & 0 & 0.06 & 0 & 0 \\
\hline$c 20: 5 n-3$ & 0 & 0 & 0.15 & 0 & 0 \\
\hline c22:5 n-3 & 0 & 0 & 2.36 & 0 & 0 \\
\hline$c 22: 6 n-3$ & 0 & 0 & 5.80 & 0 & 0 \\
\hline Total PUFA & 29.30 & 17.04 & 23.07 & 69.45 & 69.63 \\
\hline $\begin{array}{l}\text { Total } n-6 \\
\text { PUFA }\end{array}$ & 19.54 & 14.74 & 12.80 & 37.47 & 69.34 \\
\hline $\begin{array}{l}\text { Total } n-3 \\
\text { PUFA }\end{array}$ & 9.76 & 2.30 & 10.27 & 31.98 & 0.29 \\
\hline
\end{tabular}

${ }^{a}$ The values are \% of total fatty acids. The daily $3000 \mathrm{kcal}$ diet block contained $60 \mathrm{~g}$ of treatment oil. Canola, conventional canola oil; CanolaOleic, high oleic canola oil; CanolaDHA, high oleic canola and DHA oil blend; FlaxSaff, flax and safflower oil blend; CornSaff, corn and safflower oil blend; SFA, saturated fatty acids; MUFA, monounsaturated fatty acids; PUFA, polyunsaturated fatty acids.

Endothelial function testing was carried out after a 12 hour fast in the morning before breakfast at beginning and end of each phase. Scheduling pre-menopausal women for testing within the first seven days of their cycle was attempted to avoid the impact of hormonal fluctuations on the endothelial function test. Dual energy $\mathrm{x}$-ray absorptiometry (DEXA) scanning and blood pressure recording using a digital blood pressure monitor were completed at beginning and end of each treatment. Blood pressure was recorded three times and the mean value used for data analysis. Waist circumference also was taken at the beginning and 
end of each phase. Twelve-hour fasting blood samples were collected on days 1, 2, 29 and 30 in both EDTA or heparin coated tubes. Blood samples obtained on days 1 and 2 were used to determine baseline values for study endpoints, whereas blood samples obtained on the two last days were used for the endpoint values. Blood was centrifuged for 20 minutes at $520 \times \mathrm{g}$ to separate either serum or plasma depending on the requirement of the analysis. Aliquots of plasma and sera were stored at $-80^{\circ} \mathrm{C}$ until analysis. Deuterium oxide water was administered on day 29 (0.7 $\mathrm{g} \times \mathrm{kg}$ body water) to track ALA interconversion to longer chain n-3 PUFA.

\section{List of secondary endpoints analyzed in the COMIT} study

Secondary endpoints

- Cholesterol

- LDL

- HDL

- Triglycerides

- Blood glucose

- Apolipoprotein AI

- Apolipoprotein B

- CRP

- IL-6

- E-selectin

- ICAM-1

- VCAM-1

- Body fat\%

- Lean mass

- Fat mass

- Android to gynecoid ratio

- Conversion of ALA to EPA and DHA

- Fatty acid ethanolamine metabolites of oleic acid

- FADS and ELOVL genetic variant (SNP) analyses

- Lipoprotein particle size

- Lipoprotein cholesterol ester composition

- Body weight

- Waist circumference

- Blood pressure

LDL, low density lipoprotein; HDL, high density lipoprotein; CRP, C-reactive protein; IL-6, interleukin-6; ICAM-1, intercellular adhesion molecule-1; VCAM-1, vascular cell adhesion molecule-1; ALA, $\alpha$-linolenic acid; EPA, eicosapentaenoic acid; DHA, docosahexaenoic acid; FADS, fatty acid desaturase; ELOVL, elongases of very long chain fatty acids; SNP, single nucleotide polymorphism.

Aliquots of $1 \mathrm{~mm}$ plasma EDTA samples obtained from all participants on days 29 and 30 of each phase were assembled at RCFFN for total plasma fatty acid profile analyses. Given the double-blinded crossover design, compliance was assessed by total plasma fatty acid concentrations across the three clinical sites and all five phases. Plasma fatty acids were extracted by the classic Folch method using chloroform-methanol (2:1, volume to volume (v/v)) [33] containing $0.01 \%$ butylated hydroxytoluene (Sigma-Aldrich, Oakville, Ontario, Canada), followed by methylation with methanolic $\mathrm{HCl}$. Fatty acid methyl esters were then analyzed using an Agilent 6890 $\mathrm{N}$ gas chromatograph equipped with a flame ionization detector (Agilent Technologies, Mississauga, Ontario, Canada). During the extraction and methylation, heptadecanoic acid (C17:0) as an internal standard (SigmaAldrich, Oakville, Ontario, Canada). Known fatty acid standards (GLC-461, NuChek Prep, Inc. Elysian, Minnesota, United States) were used to identify the individual fatty acids in plasma samples. The level of each fatty acid was then calculated according to the corresponding peak area relative to the total area of total interested fatty acids, and considered as a percentage of the total fatty acids.

\section{Data management and statistical analysis}

A sample size of 40 subjects per each of the three centers (120 in total) was determined based on endothelial function as the primary outcome. Endothelial function was intended to be assessed by flow mediated dilatation (FMD) The anticipated difference in means was $20 \%$ and the standard deviation was 1.35 [34]. Aforementioned sample size was sufficient to detect this difference with a power of 0.8 at a significance of 0.05 . Endothelial function is a continuous variable which measures endothelium-dependent vasodilatation. Just before the beginning of the trial, it was decided to use the Endo-PAT technique for the measurement of the endothelial function, and a new sample size calculation was not made since reactive hyperemia index (RHI) measured by the Endo-PAT technique is significantly correlated with FMD [35] and has a similar variability [36]. We hypothesized that the favorable lipid changes associated with our proposed dietary interventions would improve endothelial function short-term. One hundred and seventy subjects were recruited with the anticipation of approximately a $20 \%$ dropout rate, based on our prior experience and considering the long-term commitment needed for the trial. Data from all centers were uploaded to a secure file storage system and specific centers were selected for analysis based on their expertise. Data were manually checked for accuracy. A per protocol approach with a mixed model analysis was decided as appropriate for data analysis utilizing SAS version 9.2. Kolmogorov-Smirnov (SAS version 9.2) or Cramer-von Mises (SAS version 9.2) tests and histograms were used to test the Gaussian nature of the variables and log transformation was used if necessary. 


\section{Results}

Recruitment, screening, enrollment and retention

From the methodologies used to recruit participants (Table 1), the most successful recruitment strategy at the Winnipeg center was direct mailings in selected neighborhoods. Other centers used University email list-serves to recruit participants. Figure 1 presents the flow of participants through the study. After the initial contact, $63 \%$ of volunteers were screened for clinical assessment. Only $24 \%$ of the volunteers who underwent clinical assessment were eligible for the study. A total of 170 men and women were randomized, which represents $15 \%$ of the total number who underwent primary screening. The enrollment rate was $17 \%, 18 \%$ and $12 \%$ for the Winnipeg, Québec City and University Park centers, respectively.

As shown in Figure 1, Winnipeg had the highest number of participants $(n=69)$, while University Park had the fewest $(n=43)$. The dropout rates in the centers were 22\%, 21\%, and 30\% for Winnipeg, Québec City and University Park, respectively. The overall dropout rate was $23.5 \%$. The effect of the dropouts on the study was minimal because $77.5 \%$ of the dropouts occurred within the first two phases of the study. Moreover, baseline characteristics of the dropouts and the completers did not differ significantly (data not shown). Challenges in retaining participants were expected given the nine-month duration of the trial and participant study requirements (frequent visits to the centers, as well as the full-feeding protocol). As presented in Figure 1, health issues, moving out of town, level of commitment and job issues were the major factors behind cessation in the Winnipeg and Québec City centers. Food issues and academic or work issues were the major reasons for the University Park center.

\section{Participant characteristics}

Participants were randomized to dietary treatments from September 2010 through to March 2012. Participants were classified as having metabolic syndrome as defined by IDF (Table 3 ). Winnipeg had the highest percentage of participants with metabolic syndrome (56\%), followed by University Park (43\%) and Québec City (39\%). Overall, $47 \%$ of participants were characterized as having metabolic syndrome. Although almost $50 \%$ of the participants had metabolic syndrome, their 10-year coronary heart disease risk score was less than $4 \%$ as determined by Framingham risk score calculation (data not shown). Baseline characteristics of participants from the different centers and the entire cohort are presented in Table 4.

\section{Post-treatment fatty acid profiles}

Total plasma fatty acid profiles of the completers of all five phases are summarized in Table 5. All fatty acid concentrations were calculated as percentage values of
Table 3 The number of metabolic syndrome criteria met by the participants in the COMIT study at each participating center $^{a}$

\begin{tabular}{lcccc}
\hline & Winnipeg & Québec city & University Park & Total \\
\hline One & $13.0 \%$ & $6.5 \%$ & $0 \%$ & $7.7 \%$ \\
Two & $31.5 \%$ & $54.4 \%$ & $56.7 \%$ & $45.4 \%$ \\
Three & $16.7 \%$ & $26.1 \%$ & $30.0 \%$ & $23.1 \%$ \\
Four & $33.3 \%$ & $10.9 \%$ & $13.3 \%$ & $20.8 \%$ \\
Five & $5.6 \%$ & $2.2 \%$ & $0 \%$ & $3.1 \%$ \\
\hline
\end{tabular}

Based on International Diabetic Federation defined criteria for metabolic syndrome; waist circumference ( $\geq 94 \mathrm{~cm}$ for men and $\geq 80 \mathrm{~cm}$ for women), triglycerides $\geq 1.7 \mathrm{mmol} / \mathrm{L}$, high density lipoprotein $<1 \mathrm{mmol} / \mathrm{L}$ (males) or $<1.3$ $\mathrm{mmol} / \mathrm{L}$ (females), blood pressure $\geq 130 \mathrm{mmHg}$ (systolic) and/or $\geq 85 \mathrm{mmHg}$ (diastolic) and glucose $\geq 5.5 \mathrm{mmol} / \mathrm{L}$.

total identified fatty acids measured. As DHA was part of the dietary treatment, post-treatment plasma DHA values were used to evaluate the overall compliance of the intervention study. The observed significant increases in DHA concentration only following the CanolaDHA treatment indicated good compliance in all centers. As well as the shift in DHA levels, other changes also successfully reflected their dietary intake after the 28-day treatment phases. Briefly, Canola and CanolaOleic showed the highest level of MUFA contents $(P<0.05)$, since their oleic acid levels were significantly higher than other treatments. Two PUFA-rich diets significantly elevated PUFA contents compared to the other three canola-based MUFA diets $(P<0.05)$, due to their higher levels of linoleic acid. FlaxSaff provided the lowest level of arachidonic acid $(P<0.05)$, indicating the competition between $n-3$ and $n-6$ synthesis as the FlaxSaff oil treatment provided the highest level of dietary ALA. CanolaDHA and FlaxSaff also showed higher levels of EPA compared to other three treatments $(P<0.05)$. In general, the ratio of n- 6 to $n-3$ also successfully explained the corresponding response to $n-3$ rich and $n-6$ rich dietary treatments, indicating good compliance among participants across the entire study.

\section{Primary and secondary outcome measures}

Data analyses of endothelial function and secondary outcome measures given in "List of secondary endpoints analyzed in the COMIT study" section are currently being carried out.

\section{Adverse effects}

Adverse effects were monitored using a questionnaire distributed by study staff on every Friday of each dietary phase. Additionally, the clinical study staff assessed the tolerability of the treatment each day when they interacted with the participants. No adverse effects of any treatment diets were reported. 
Table 4 Baseline characteristics of participants at each participating center

\begin{tabular}{|c|c|c|c|c|c|}
\hline & Winnipeg & Québec city & University Park & Total & $P$ value $^{\mathrm{a}}$ \\
\hline Male & 16 & 29 & 15 & 60 & \\
\hline Female & 38 & 17 & 15 & 70 & 0.003 \\
\hline BMI $\left(\mathrm{kg} / \mathrm{m}^{2}\right)$ & $29.7 \pm 5.0$ & $30.0 \pm 4.3$ & $29.7 \pm 3.3$ & $29.8 \pm 4.4$ & 0.928 \\
\hline Age (years) & $43.9 \pm 15.8$ & $49.9 \pm 14.2$ & $45.9 \pm 9.6$ & $46.5 \pm 14.2$ & 0.105 \\
\hline Total cholesterol (mmol/L) & $5.4 \pm 1.1$ & $5.4 \pm 1.0$ & $5.1 \pm 0.9$ & $5.3 \pm 1.1$ & 0.371 \\
\hline $\mathrm{HDL}(\mathrm{mmol} / \mathrm{L})$ female & $1.3 \pm 0.1$ & $1.4 \pm 0.1$ & $1.3 \pm 0.1$ & $1.3 \pm 0.1$ & 0.413 \\
\hline male & $1.1 \pm 0.1$ & $1.1 \pm 0.1$ & $1.1 \pm 0.1$ & $1.1 \pm 0.1$ & 0.906 \\
\hline LDL (mmol/L) & $3.4 \pm 1.0$ & $3.4 \pm 0.9$ & $3.2 \pm 0.9$ & $3.4 \pm 0.9$ & 0.572 \\
\hline Triglycerides (mmol/L) & $1.6 \pm 0.9$ & $1.8 \pm 0.8$ & $1.5 \pm 0.9$ & $1.7 \pm 0.9$ & 0.338 \\
\hline Glucose (mmol/l) & $5.4 \pm 1.7$ & $5.3 \pm 0.6$ & $5.3 \pm 0.5$ & $5.4 \pm 1.1$ & 0.788 \\
\hline Body weight (kg) & $80.7 \pm 15.9$ & $85.8 \pm 15.3$ & $89.9 \pm 15.7$ & $84.7 \pm 15.9$ & 0.042 \\
\hline Waist $(\mathrm{cm})$ female & $90.6 \pm 11.9$ & $97.4 \pm 10.3$ & $98.5 \pm 9.9$ & $93.4 \pm 11.6$ & 0.033 \\
\hline male & $103.4 \pm 13.8$ & $106.9 \pm 8.7$ & $106.5 \pm 6.5$ & $105.8 \pm 9.9$ & 0.508 \\
\hline SBP $(\mathrm{mmHg})$ & $122.4 \pm 20.3$ & $119.8 \pm 14.2$ & $118.5 \pm 12.4$ & $120.6 \pm 16.7$ & 0.548 \\
\hline DBP (mmHg) & $81.2 \pm 12.4$ & $70.5 \pm 10.5$ & $79.7 \pm 7.40$ & $77.0 \pm 11.8$ & $<0.001$ \\
\hline
\end{tabular}

${ }^{a}$ Analysis of variance was used to analyze between-group differences in continuous variables. For categorical variables, Fisher's exact test or chi square test was used. BMI, body mass index; HDL, high density lipoprotein; LDL, low density lipoprotein; SBP, systolic blood pressure; DBP, diastolic blood pressure.

\section{Discussion}

The recruitment and retention strategies employed resulted in the successful completion of the required number of participants. Retention was also high due to the dedication and commitment of the study volunteers and clinical coordinators, as well as the success of the social retention enhancement program. Differences across centers in demographic characteristics and baseline parameters were limited to the male to female ratio, body weight, waist circumference in females, and diastolic blood pressure. The success rate of participant completion was numerically close between Winnipeg and Québec City, with the University Park center having a lower rate. The major reason for dropouts in Winnipeg and Québec City were health reasons, while in University Park it was more related to experimental diet acceptability.

Predictable elevations of specific plasma fatty acids representing the major dietary fatty acids in the intervention oils were observed as expected. For example, the three-fold elevation of plasma ALA following the FlaxSaff dietary phase compared to the CornSaff dietary phase demonstrated the ability of flax oil-enriched shakes to raise serum ALA levels. Similarly, the 1.3-fold elevation of oleic acid after the CanolaOleic treatment compared to CornSaff treatment is consistent with its high oleic acid content. Significantly higher eicosapentaenoic acid (EPA) levels in Canola and FlaxSaff compared to other treatments are evidence for the metabolic conversion of ALA to EPA. The significant elevation of EPA in CanolaDHA compared to other treatments (except FlaxSaff) could probably be due to the higher EPA content in the algal sourced DHA that was used in this study. However DHA might convert back to EPA in CanolaDHA, which can be further confirmed by the stable isotope-trafficking assays in this study $[37,38]$. Similarly, the more than two-fold elevation of DHA after the CanolaDHA treatment, compared to other treatments, is not surprising and is in line with previous reports [39-41]. Lack of a DHA raising effect of FlaxSaff treatment agrees with prior work demonstrating low efficiency of conversion of ALA to DHA [31]. Remarkable consistency was observed in compliance among participants as depicted here by predictable shifts in their fatty acid profile at the end of each dietary period, highlighting the successful completion of this long-term full-feeding clinical trial.

Multicenter randomized controlled clinical trials exist as the gold standard for evidence-based research [42]. The multicenter nature of the trial ensured the robustness of results across different centers. The iso-caloric, weightmaintaining, full-feeding regimen eliminated the effect of variable habitual, background diets on study outcomes. Caloric excess or restriction can potentially affect blood lipids, and even body composition and body weight loss can affect endothelial function [27], hence requiring standardized study conditions to prevent weight change. Study treatments oils were blended at the RCFFN and shipped to participating centers, removing an important source of variation. Sample analysis for specific endpoints was completed for all samples at the center designated for a particular analysis, in order to remove the effect of interlaboratory variation. Clinical measurement protocols, particularly for endothelial function testing, were harmonized across centers by training personnel from the University Park center staff. The crossover design eliminated the 
Table 5 Plasma fatty acid profile of participants at the end of each dietary phase in 130 participants $(\mathrm{g} / 100 \mathrm{~g})^{\mathrm{a}}$

\begin{tabular}{|c|c|c|c|c|c|}
\hline & Canola & CanolaOleic & CanolaDHA & FlaxSaff & CornSaff \\
\hline c14:0 & $0.71 \pm 0.04$ & $0.74 \pm 0.04$ & $0.71 \pm 0.04$ & $0.72 \pm 0.04$ & $0.68 \pm 0.04$ \\
\hline c14:1 & $0.12 \pm 0.02$ & $0.08 \pm 0.02$ & $0.09 \pm 0.02$ & $0.08 \pm 0.02$ & $0.07 \pm 0.02$ \\
\hline c16:0 & $27.04 \pm 0.20^{\mathrm{a}}$ & $27.40 \pm 0.20^{\mathrm{a}}$ & $28.10 \pm 0.20^{b}$ & $27.41 \pm 0.20^{\mathrm{a}}$ & $27.35 \pm 0.20^{\mathrm{a}}$ \\
\hline c16:1 & $1.14 \pm 0.08$ & $1.10 \pm 0.08$ & $0.94 \pm 0.08$ & $1.30 \pm 0.08$ & $1.13 \pm 0.75$ \\
\hline c18:0 & $11.83 \pm 0.10^{a}$ & $11.79 \pm 0.10^{\mathrm{a}}$ & $12.28 \pm 0.10^{b}$ & $12.51 \pm 0.10^{c}$ & $12.34 \pm 0.10^{b c}$ \\
\hline c18:1 & $14.90 \pm 0.19^{a}$ & $15.52 \pm 0.19^{b}$ & $13.36 \pm 0.19^{c}$ & $12.10 \pm 0.18^{d}$ & $11.62 \pm 0.18^{d}$ \\
\hline c18:2 n-6 & $22.00 \pm 0.23^{a}$ & $21.52 \pm 0.23^{a}$ & $18.68 \pm 0.23^{b}$ & $25.13 \pm 0.23^{c}$ & $25.93 \pm 0.23^{d}$ \\
\hline c18:3 n-6 & $0.17 \pm 0.07$ & $0.18 \pm 0.07$ & $0.23 \pm 0.07$ & $0.12 \pm 0.07$ & $0.17 \pm 0.07$ \\
\hline c18:3 n-3 & $0.79 \pm 0.03^{a}$ & $0.63 \pm 0.03^{b}$ & $0.57 \pm 0.03^{b c}$ & $1.61 \pm 0.03^{c}$ & $0.49 \pm 0.03^{d}$ \\
\hline c20:0 & $0.50 \pm 0.02^{\mathrm{a}}$ & $0.48 \pm 0.02^{\mathrm{ab}}$ & $0.49 \pm 0.02^{\mathrm{a}}$ & $0.41 \pm 0.02^{b}$ & $0.46 \pm 0.02^{\mathrm{ab}}$ \\
\hline c20:1 & $0.32 \pm 0.02^{a}$ & $0.32 \pm 0.02^{\mathrm{a}}$ & $0.27 \pm 0.02^{\mathrm{ab}}$ & $0.18 \pm 0.02^{c}$ & $0.23 \pm 0.02^{b c}$ \\
\hline$c 20: 2 n-6$ & $0.35 \pm 0.03^{\mathrm{a}}$ & $0.31 \pm 0.03^{\mathrm{ab}}$ & $0.24 \pm 0.03^{b}$ & $0.32 \pm 0.03^{\mathrm{ab}}$ & $0.37 \pm 0.03^{\mathrm{a}}$ \\
\hline$c 20: 3 n-6$ & $2.45 \pm 0.07^{\mathrm{ab}}$ & $2.53 \pm 0.07^{b}$ & $1.79 \pm 0.07^{c}$ & $1.86 \pm 0.07^{c}$ & $2.28 \pm 0.07^{\mathrm{a}}$ \\
\hline c20:4 n-6 & $9.28 \pm 0.15^{a}$ & $9.67 \pm 0.15^{b}$ & $9.70 \pm 0.15^{b}$ & $8.27 \pm 0.15^{c}$ & $9.59 \pm 0.15^{\mathrm{ab}}$ \\
\hline c20:5 n-3 & $1.09 \pm 0.04^{\mathrm{a}}$ & $0.86 \pm 0.04^{b}$ & $1.53 \pm 0.04^{c}$ & $1.45 \pm 0.04^{c}$ & $0.49 \pm 0.04^{d}$ \\
\hline c22:0 & $0.94 \pm 0.03^{\mathrm{ab}}$ & $0.90 \pm 0.03^{\mathrm{ab}}$ & $1.00 \pm 0.03^{\mathrm{a}}$ & $0.90 \pm 0.03^{b}$ & $0.93 \pm 0.03^{b}$ \\
\hline c22:4 n-6 & $0.43 \pm 0.08$ & $0.29 \pm 0.08$ & $0.25 \pm 0.08$ & $0.22 \pm 0.08$ & $0.39 \pm 0.08$ \\
\hline c22:5 n-3 & $0.81 \pm 0.03^{a}$ & $0.72 \pm 0.03^{b}$ & $0.34 \pm 0.03^{c}$ & $0.97 \pm 0.03^{d}$ & $0.62 \pm 0.03^{e}$ \\
\hline$c 22: 6 n-3$ & $2.84 \pm 0.09^{a}$ & $2.79 \pm 0.09^{a}$ & $7.21 \pm 0.10^{b}$ & $2.59 \pm 0.09^{a}$ & $2.66 \pm 0.09^{a}$ \\
\hline c24:0 & $0.72 \pm 0.03^{\mathrm{a}}$ & $0.65 \pm 0.03^{b}$ & $0.74 \pm 0.03^{a}$ & $0.74 \pm 0.03^{\mathrm{a}}$ & $0.73 \pm 0.03^{a}$ \\
\hline c24:1 & $1.56 \pm 0.05^{\mathrm{a}}$ & $1.49 \pm 0.05^{\mathrm{ab}}$ & $1.55 \pm 0.05^{a}$ & $1.38 \pm 0.05^{b}$ & $1.44 \pm 0.05^{\mathrm{ab}}$ \\
\hline Total SFA & $41.74 \pm 0.19^{\mathrm{a}}$ & $41.92 \pm 0.19^{\mathrm{ab}}$ & $43.28 \pm 0.19^{c}$ & $42.71 \pm 0.19^{c d}$ & $42.51 \pm 0.19^{b c}$ \\
\hline Total MUFA & $18.05 \pm 0.22^{\mathrm{a}}$ & $18.50 \pm 0.22^{\mathrm{a}}$ & $16.20 \pm 0.22^{b}$ & $14.78 \pm 0.22^{c}$ & $14.49 \pm 0.22^{c}$ \\
\hline Total PUFA & $40.21 \pm 0.22^{\mathrm{a}}$ & $39.54 \pm 0.22^{b}$ & $40.47 \pm 0.22^{a}$ & $42.57 \pm 0.22^{c}$ & $43.04 \pm 0.22^{c}$ \\
\hline Total n-6 PUFA & $34.67 \pm 0.23^{a}$ & $34.46 \pm 0.23^{\mathrm{a}}$ & $30.92 \pm 0.23^{b}$ & $35.92 \pm 0.23^{c}$ & $38.79 \pm 0.23^{d}$ \\
\hline Total n-3 PUFA & $5.53 \pm 0.10^{\mathrm{a}}$ & $5.03 \pm 0.10^{b}$ & $9.62 \pm 0.11^{c}$ & $6.64 \pm 0.10^{d}$ & $4.24 \pm 0.10^{e}$ \\
\hline$n-6: n-3$ & $6.55 \pm 0.13^{\mathrm{a}}$ & $7.18 \pm 0.14^{b}$ & $3.32 \pm 0.14^{c}$ & $5.65 \pm 0.13^{d}$ & $9.41 \pm 0.13^{e}$ \\
\hline
\end{tabular}

${ }^{a}$ The values are $\%$ abundance of each fatty acid to total fatty acids given as least squares mean \pm SE for 130 individuals. Total fatty acid values and ratios are calculated. Mixed-effects repeated-measures analysis of variance with treatment, age, gender and the dietary phase as fixed effect, the last diet (sequence effect) as a random effect, and the measures for each subject by phases were used for the data analysis. Canola, conventional canola oil; CanolaOleic, high oleic canola oil; CanolaDHA, high oleic canola and DHA oil blend; FlaxSaff, flax and safflower oil blend; CornSaff, corn and safflower oil blend; SFA, saturated fatty acids; MUFA, monounsaturated fatty acids; PUFA, polyunsaturated fatty acids. ${ }^{\text {abcde }}$ Mean values with different superscript letters across rows denote significant differences at $P<0.05$.

effect of individual differences on response to treatments and reduced the required sample size. Endothelial function was found to be significantly changed in studies with a crossover or a parallel design over a period of four weeks following dietary intervention, indicating that the study design and the duration of the current study is appropriate for detecting changes in endothelial function [43,44]. Moreover, an acute study that measured endothelial function by FMD four hours after a high-fat meal supplemented with either $25 \mathrm{~g}$ olive oil or $40 \mathrm{~g}$ of shelled walnut in hypercholesterolemic subjects [34] also showed significant changes. FMD [45] and Endo-PAT techniques [46] are both dependent on nitric oxide mediated vasodilatation which is affected by both short- and long-term changes in endothelial function. Even though the measure of percent change in endothelial function has no clinical relevance as yet, a lower range of endothelial function (lower two tertiles) as measured by FMD, is associated with enhanced risk of future cardiovascular events [47].

The final sample size of 130 allowed us to detect a difference of $9.4 \%$ or greater in the RHI, which is a measure of the endothelial function, and a $10 \%$ difference of LDL-C with a power of 0.8 at a significance of 0.05 .

Diets were designed to address contemporary nutrition questions. Although MUFA generally decreases TC and LDL-C, the efficacy is considered lesser than PUFA $[48,49]$. However, the relative benefits of individual unsaturated fatty acid classes are increasingly being challenged; this study was designed to resolve some of these issues. 
The primary and ancillary measurements in our study will link the changes in surrogate cardiovascular biomarkers such as blood lipids and glucose, inflammatory biomarkers and hormones, to clinical endpoints such as endothelial function and body fat composition. The mechanistic studies, including lipid trafficking, apolipoproteins, lipoprotein particle size, and reverse cholesterol transport, will assist in clarifying the relationship between the biomarkers and clinical endpoints. Finally, results will be evaluated from the perspective of genetic variation, assessed through single nucleotide polymorphisms among individuals to assess why biomarker responses vary as a function of diet treatments.

The study possesses certain limitations. The disparity in male: female ratio and the baseline differences in cholesterol across centers can be considered as confounding treatment effects. However, use of a mixed model approach in statistical analyses is expected to minimize this effect. The effect of the preceding diet or the sequence of treatment is another confounding factor that was anticipated to be minimized by the mixed model approach. Moreover, differences in bioactives other than fatty acid composition within the treatment oils might affect clinical measurements such as endothelial function. The intraindividual and inter-operator variability in endothelial function testing might likewise obscure a treatment effect. Thus, standardization of the test procedure represents an important factor in maintaining the robustness of endothelial function data [50]. Steps were taken to limit this variability by controlling the environment and also by minimizing any additional factors such as the stage in the menstrual cycle of female participants [51], medication, and tea or coffee drinking [52]. Additionally, use of the corn and safflower oil blend as the alternate treatment would be expected to improve blood lipids and other parameters [6]. Therefore, it may not be possible to see an improvement in blood lipids in certain treatments compared to the corn and safflower oil blend treatment.

\section{Conclusions}

The successful completion of 130 participants through the study protocol of this nine-month long, full-feeding, multicenter study represents a significant achievement. Recruitment and retention strategies used in this study were instrumental in achieving this goal. Standardized protocols and efforts to minimize variation are expected to lead to findings that will help to fill the gaps in our knowledge of the metabolism, lipid trafficking, and clinical efficacy of the major classes of dietary fatty acids.

\section{Trial status}

The trial started recruiting volunteers in September 2010 and completed the intervention stage in March 2012.

\section{Competing interests}

PJJ reported receiving grants from Advanced Foods and Materials Network (AFM Net), Danone, Enzymotec, Unilever, the Canadian Institutes of Health Research (CIHR) and Canada Research Chair Endowment (CRCE) of the Federal Government of Canada. PJJ also serves as President of Nutritional Fundamentals for Health Inc, which markets plant sterols among other nutraceuticals. BL has received research grants from the Dairy Farmers of Canada, Dairy Australia, Danone Institute and Atrium Innovations, and honoraria from Unilever, Danone, and the Dairy Farmers of Canada. BL is Chair in Nutrition and Cardiovascular Health, supported in part by Provigo/Loblaws. DAJ reported serving on the Scientific Advisory Board of Unilever, Sanitarium Company, California Strawberry Commission, Loblaw Supermarket, Herbal Life International, Nutritional Fundamental for Health, Pacific Health Laboratories, Metagenics, Bayer Consumer Care, Orafti, Dean Foods, Kellogg's, Quaker Oats, Procter \& Gamble, Coca-Cola, NuVal Griffin Hospital, Abbott, Pulse Canada, Saskatchewan Pulse Growers, and Canola Council of Canada; receiving honoraria for scientific advice from the Almond Board of California, International Tree Nut Council Nutrition Research and Education Foundation, Barilla, Unilever Canada, Solae, Oldways, Kellogg's, Quaker Oats, Procter \& Gamble, Coca-Cola, NuVal Griffin Hospital, Abbott, Canola Council of Canada, Dean Foods, California Strawberry Commission, Haine Celestial, and Alpro Foundation; being on the speakers panel for the Almond Board of California; receiving research grants from Loblaw Brands Ltd, Unilever, Barilla, Almond Board of California, Solae, Haine Celestial, Sanitarium Company, Orafti, International Tree Nut Council, and Peanut Institute; and receiving travel support to meetings from the Almond Board of California, Unilever, Alpro Foundation, and International Tree Nut Council, Canadian Institutes for Health Research, Canada Foundation for Innovation, Ontario Research Fund. DAJ receives salary support as a Canada Research Chair from the federal government of Canada. DAJ's wife is a director of Glycemic Index Laboratories, Toronto, Ontario, Canada. SGW has received research funding and consulting fees from the Canola Council of Canada and Flax Canada 2013. PMKE serves on the Unilever Scientific Advisory Board. Other authors did not report any conflicts of financial interests.

\section{Authors' contributions}

PJJ is responsible for the integrity and the accuracy of the data and had full access to the complete data set in the study. Study concept and design: PJJ, DAJ, BL, SGW and PMKE. Acquisition of data: PJJ, DAJ, BL, SGW, PMKE, VKS, SP, $J A F, X L$ and CEM, Analysis and interpretation of data: PJJ, VKS and SP. Drafting of the manuscript: VKS, SP, and PJJ. Critical revision of the manuscript for important intellectual content and final approval of the manuscript: PJJ, DAJ, BL, SGW and PMKE. Obtained funding: PJJ, DAJ, BL, SGW and PMKE. Study supervision: PJJ, DAJ, BL, SGW and PMKE. All authors reviewed the manuscript. All authors read and approved the final manuscript.

\section{Acknowledgements}

Canola Council of Canada, Flax Council of Canada, Agriculture and Agri-Food Canada, Dow Agrosciences, Canada Research Chairs, and Western Grains Research Foundation collectively funded this study through the Growing Forward program of Agriculture and Agri-Food Canada. The project described was also supported by the National Center for Research Resources, Grant UL1 RR033184, and is now at the National Center for Advancing Translational Sciences, Grant UL1 TR000127. The content is solely the responsibility of the authors and does not necessarily represent the official views of the $\mathrm{NIH}$. The authors acknowledge Dr Todd Rideout, assistant professor at University of Buffalo for contribution to the initial clinical trial design. Dr Rasheda Rabbani, biostatistician at Manitoba Institute of Child Health is kindly acknowledged for statistical analysis of data. Funders did not participate in the design, conduct, management, data collection and analysis or preparation and review of the manuscript.

\section{Author details}

${ }^{1}$ Richardson Centre for Functional Foods and Nutraceuticals, University of Manitoba, 196 Innovation Drive, SmartPark, Winnipeg, MB R3T 6C5, Canada. ${ }^{2}$ St. Michaels Hospital, 30 Bond Street, Toronto, ON M5B 1W8, Canada. ${ }^{3}$ Institute of Nutrition and Functional Foods, Pavillon des Services, Université Laval, Suite 1705, 2440 Hochelaga Blvd, Québec, QC G1V 0A6, Canada. ${ }^{4}$ Department of Nutritional Sciences, Pennsylvania State University, 110 Chandlee Laboratory, University Park, PA 16802, USA. ${ }^{5}$ Department of Biobehavioral Health, Pennsylvania State University, 219 Biobehavioral Health 
Building, University Park, PA 16802, USA. ${ }^{6}$ Current address: Phenomenome Discoveries Inc, Saskatoon, Saskatchewan, Canada.

Received: 9 August 2013 Accepted: 1 April 2014 Published: 23 April 2014

\section{References}

1. Slattery ML, Randall DE: Trends in coronary heart disease mortality and food consumption in the United States between 1909 and 1980. Am J Clin Nutr 1988, 47:1060-1067.

2. McGuire S: U.S. Department of Agriculture and U.S. Department of Health and Human Services, Dietary Guidelines for Americans, 2010. 7th Edition, Washington, DC: U.S. Government Printing Office, January 2011. Adv Nutr 2011, 2:293-294.

3. Rasmussen BM, Vessby B, Uusitupa M, Berglund L, Pedersen E, Riccardi G, Rivellese AA, Tapsell L, Hermansen K: Effects of dietary saturated, monounsaturated, and $n-3$ fatty acids on blood pressure in healthy subjects. Am J Clin Nutr 2006, 83:221-226

4. Mensink RP, Katan MB: Effect of dietary fatty acids on serum lipids and lipoproteins. A meta-analysis of 27 trials. Arterioscler Thromb 1992, 12:911-919.

5. Rivellese AA, Maffettone A, Vessby B, Uusitupa M, Hermansen K, Berglund L, Louheranta A, Meyer BJ, Riccardi G: Effects of dietary saturated, monounsaturated and $\mathrm{n}-3$ fatty acids on fasting lipoproteins, LDL size and post-prandial lipid metabolism in healthy subjects. Atherosclerosis 2003 167:149-158.

6. Bjermo H, Iggman D, Kullberg J, Dahlman I, Johansson L, Persson L, Berglund J, Pulkki K, Basu S, Uusitupa M, Rudling M, Arner P, Cederholm T, Ahlström H, Risérus U: Effects of n-6 PUFAs compared with SFAs on liver fat, lipoproteins, and inflammation in abdominal obesity: a randomized controlled trial. Am J Clin Nutr 2012, 95:1003-1012.

7. Hodson L, Skeaff CM, Chisholm WA: The effect of replacing dietary saturated fat with polyunsaturated or monounsaturated fat on plasma lipids in free-living young adults. Eur J Clin Nutr 2001, 55:908-915.

8. Hunter JE, Zhang J, Kris-Etherton PM: Cardiovascular disease risk of dietary stearic acid compared with trans, other saturated, and unsaturated fatty acids: a systematic review. Am J Clin Nutr 2010, 91:46-63.

9. Buhr KA: Surrogate end points in secondary analyses of cardiovascular trials. Prog Cardiovasc Dis 2012, 54:343-350.

10. Baylin A, Ruiz-Narvaez E, Kraft P, Campos H: Alpha-Linolenic acid, Delta6-desaturase gene polymorphism, and the risk of nonfatal myocardial infarction. Am J Clin Nutr 2007, 85:554-560.

11. Hussein N, Ah-Sing E, Wilkinson P, Leach C, Griffin BA, Millward DJ: Long-chain conversion of [13C]linoleic acid and alpha-linolenic acid in response to marked changes in their dietary intake in men. J Lipid Res 2005, 46:269-280.

12. Egert S, Somoza V, Kannenberg F, Fobker M, Krome K, Erbersdobler HF, Wahrburg U: Influence of three rapeseed oil-rich diets, fortified with alpha-linolenic acid, eicosapentaenoic acid or docosahexaenoic acid on the composition and oxidizability of low-density lipoproteins: results of a controlled study in healthy volunteers. Eur J Clin Nutr 2007, 61:314-325.

13. Ramsden CE, Hibbeln JR, Majchrzak SF, Davis JM: $n-6$ fatty acid-specific and mixed polyunsaturate dietary interventions have different effects on CHD risk: a meta-analysis of randomised controlled trials. Br J Nutr 2010, 104:1586-1600

14. Yashodhara BM, Umakanth S, Pappachan JM, Bhat SK, Kamath R, Choo BH: Omega-3 fatty acids: a comprehensive review of their role in health and disease. Postgrad Med J 2009, 85:84-90.

15. Pischon T, Hankinson SE, Hotamisligil GS, Rifai N, Willett WC, Rimm EB: Habitual dietary intake of $n-3$ and $n-6$ fatty acids in relation to inflammatory markers among US men and women. Circulation 2003, 108:155-160.

16. Ramsden CE, Zamora D, Leelarthaepin B, Majchrzak-Hong SF, Faurot KR, Suchindran CM, Ringel A, Davis JM, Hibbeln JR: Use of dietary linoleic acid for secondary prevention of coronary heart disease and death: evaluation of recovered data from the Sydney Diet Heart Study and updated meta-analysis. BMJ 2013, 346:e8707.

17. Stenvinkel P: Endothelial dysfunction and inflammation-is there a link? Nephrol Dial Transplant 2001, 16:1968-1971.

18. van Bussel BC, Soedamah-Muthu SS, Henry RM, Schalkwijk CG, Ferreira I, Chaturvedi N, Toeller M, Fuller JH, Stehouwer CD: Unhealthy dietary patterns associated with inflammation and endothelial dysfunction in type 1 diabetes: The EURODIAB study. Nutr Metab Cardiovasc Dis 2013, 23:758-64.

19. Duvnjak L, Duvnjak M: The metabolic syndrome - an ongoing story. J Physiol Pharmacol 2009, 60(Suppl 7):19-24.

20. Alberti KG, Eckel RH, Grundy SM, Zimmet PZ, Cleeman Jl, Donato KA, Fruchart JC, James WP, Loria CM, Smith SC Jr: Harmonizing the metabolic syndrome: a joint interim statement of the International Diabetes Federation Task Force on Epidemiology and Prevention; National Heart Lung, and Blood Institute; American Heart Association; World Heart Federation; International Atherosclerosis Society; and International Association for the Study of Obesity. Circulation 2009, 120:1640-1645.

21. Potenza MV, Mechanick Jl: The metabolic syndrome: definition, global impact, and pathophysiology. Nutr Clin Pract 2009, 24:560-577.

22. Muoio DM, Newgard CB: Obesity-related derangements in metabolic regulation. Annu Rev Biochem 2006, 75:367-401.

23. Gillingham $L G$, Robinson $\mathrm{KS}$, Jones PJ: Effect of high-oleic canola and flaxseed oils on energy expenditure and body composition in hypercholesterolemic subjects. Metabolism 2012, 61:1598-1605.

24. Due A, Larsen TM, Hermansen K, Stender S, Holst JJ, Toubro S, Martinussen T, Astrup A: Comparison of the effects on insulin resistance and glucose tolerance of 6-mo high-monounsaturated-fat, low-fat, and control diets. Am J Clin Nutr 2008, 87:855-862.

25. Buckley JD, Howe PR: Anti-obesity effects of long-chain omega-3 polyunsaturated fatty acids. Obes Rev 2009, 10:648-659.

26. Martinez-Victoria E, Yago MD: Omega 3 polyunsaturated fatty acids and body weight. Br J Nutr 2012, 107(Suppl 2):S107-116.

27. Merino J, Megias-Rangil I, Ferre R, Plana N, Girona J, Rabasa A, Aragones G, Cabre A, Bonada A, Heras M, Masana L: Body weight loss by very-low-calorie diet program improves small artery reactive hyperemia in severely obese patients. Obes Surg 2013, 23:17-23.

28. Grundy SM, Brewer HB Jr, Cleeman Jl, Smith SC Jr, Lenfant C: Definition of metabolic syndrome: Report of the National Heart, Lung, and Blood Institute/American Heart Association conference on scientific issues related to definition. Circulation 2004, 109:433-438.

29. Alberti KG, Zimmet P, Shaw J: Metabolic syndrome-a new world-wide definition. A Consensus Statement from the International Diabetes Federation. Diabet Med 2006, 23:469-480

30. Mifflin MD, St Jeor ST, Hill LA, Scott BJ, Daugherty SA, Koh YO: A new predictive equation for resting energy expenditure in healthy individuals. Am J Clin Nutr 1990, 51:241-247.

31. Gillingham LG, Gustafson JA, Han SY, Jassal DS, Jones PJ: High-oleic rapeseed (canola) and flaxseed oils modulate serum lipids and inflammatory biomarkers in hypercholesterolaemic subjects. Br J Nutr 2011, 105:417-427.

32. McCrea CE, Skulas-Ray AC, Chow M, West SG: Test-retest reliability of pulse amplitude tonometry measures of vascular endothelial function: implications for clinical trial design. Vasc Med 2012, 17:29-36.

33. Folch J, Lees M, Sloane Stanley GH: A simple method for the isolation and purification of total lipides from animal tissues. J Biol Chem 1957, 226:497-509.

34. Cortes B, Nunez I, Cofan M, Gilabert R, Perez-Heras A, Casals E, Deulofeu R, Ros E: Acute effects of high-fat meals enriched with walnuts or olive oil on postprandial endothelial function. J Am Coll Cardiol 2006, 48:1666-1671

35. Kuvin JT, Patel AR, Sliney KA, Pandian NG, Sheffy J, Schnall RP, Karas RH, Udelson JE: Assessment of peripheral vascular endothelial function with finger arterial pulse wave amplitude. Am Heart J 2003, 146:168-174.

36. Liu J, Wang J, Jin Y, Roethig HJ, Unverdorben M: Variability of peripheral arterial tonometry in the measurement of endothelial function in healthy men. Clin Cardiol 2009, 32:700-704.

37. Kaur G, Begg DP, Barr D, Garg M, Cameron-Smith D, Sinclair AJ: Short-term docosapentaenoic acid (22:5 n-3) supplementation increases tissue docosapentaenoic acid, DHA and EPA concentrations in rats. Br J Nutr 2010, 103:32-37.

38. Mozaffarian $\mathrm{D}, \mathrm{Wu} \mathrm{JH}:(\mathrm{n}-3)$ fatty acids and cardiovascular health: are effects of EPA and DHA shared or complementary? I Nutr 2012, 142:614S-625S.

39. Sagara M, Njelekela M, Teramoto T, Taguchi T, Mori M, Armitage L, Birt N Birt C, Yamori Y: Effects of docosahexaenoic Acid supplementation on blood pressure, heart rate, and serum lipids in Scottish men with hypertension and hypercholesterolemia. Int J Hypertens 2011, 2011:809198. 
40. Theobald HE, Chowienczyk PJ, Whittall R, Humphries SE, Sanders TA: LDL cholesterol-raising effect of low-dose docosahexaenoic acid in middle-aged men and women. Am J Clin Nutr 2004, 79:558-563.

41. Davidson MH, Maki KC, Kalkowski J, Schaefer EJ, Torri SA, Drennan KB: Effects of docosahexaenoic acid on serum lipoproteins in patients with combined hyperlipidemia: a randomized, double-blind, placebo-controlled trial. J Am Coll Nutr 1997, 16:236-243.

42. Nallamothu BK, Hayward RA, Bates ER: Beyond the randomized clinical trial: the role of effectiveness studies in evaluating cardiovascular therapies. Circulation 2008, 118:1294-1303.

43. Kwak JH, Paik JK, Kim HI, Kim OY, Shin DY, Kim HJ, Lee JH, Lee JH: Dietary treatment with rice containing resistant starch improves markers of endothelial function with reduction of postprandial blood glucose and oxidative stress in patients with prediabetes or newly diagnosed type 2 diabetes. Atherosclerosis 2012, 224:457-464.

44. Ros E, Nunez I, Perez-Heras A, Serra M, Gilabert R, Casals E, Deulofeu R: A walnut diet improves endothelial function in hypercholesterolemic subjects: a randomized crossover trial. Circulation 2004, 109:1609-1614.

45. Deanfield JE, Halcox JP, Rabelink TJ: Endothelial function and dysfunction: testing and clinical relevance. Circulation 2007, 115:1285-1295.

46. Hamburg NM, Benjamin EJ: Assessment of endothelial function using digital pulse amplitude tonometry. Trends Cardiovasc Med 2009, 19:6-11.

47. Gokce N, Keaney JF Jr, Hunter LM, Watkins MT, Nedeljkovic ZS, Menzoian JO, Vita JA: Predictive value of noninvasively determined endothelial dysfunction for long-term cardiovascular events in patients with peripheral vascular disease. J Am Coll Cardiol 2003, 41:1769-1775.

48. Truswell AS, Choudhury N: Monounsaturated oils do not all have the same effect on plasma cholesterol. Eur J Clin Nutr 1998, 52:312-315.

49. Adkins Y, Kelley DS: Mechanisms underlying the cardioprotective effects of omega-3 polyunsaturated fatty acids. J Nutr Biochem 2010, 21:781-792.

50. Onkelinx S, Cornelissen V, Goetschalckx K, Thomaes T, Verhamme P, Vanhees $L$ : Reproducibility of different methods to measure the endothelial function. Vasc Med 2012, 17:79-84.

51. Williams MR, Westerman RA, Kingwell BA, Paige J, Blombery PA, Sudhir K, Komesaroff PA: Variations in endothelial function and arterial compliance during the menstrual cycle. J Clin Endocrinol Metab 2001, 86:5389-5395.

52. Njike W, Faridi Z, Shuval K, Dutta S, Kay CD, West SG, Kris-Etherton PM, Katz DL: Effects of sugar-sweetened and sugar-free cocoa on endothelial function in overweight adults. Int J Cardiol 2009, 149:83-88.

doi:10.1186/1745-6215-15-136

Cite this article as: Senanayake et al:: Plasma fatty acid changes

following consumption of dietary oils containing $n-3, n-6$, and $n-9$ fatty acids at different proportions: preliminary findings of the Canola Oil Multicenter Intervention Trial (COMIT). Trials 2014 15:136.

\section{Submit your next manuscript to BioMed Central and take full advantage of:}

- Convenient online submission

- Thorough peer review

- No space constraints or color figure charges

- Immediate publication on acceptance

- Inclusion in PubMed, CAS, Scopus and Google Scholar

- Research which is freely available for redistribution 